\title{
A MULTI PHASE FIELD CONCEPT: NUMERICAL SIMULATIONS OF MOVING PHASE BOUNDARIES AND MULTIPLE JUNCTIONS
}

\author{
HARALD GARCKE, BRITTA NESTLER, AND BARBARA STOTH
}

\begin{abstract}
We present numerical simulations which support the formal asymptotic analysis relating a multi order parameter Allen-Cahn system to a multi phase interface problem with curvature dependent evolution of the interfaces and angle conditions at triple junctions. Within the gradient energy of the Allen-Cahn system, the normal to an interface between phases $i$ and $j$ is modeled by the irreducible representations $\left(u_{i} \nabla u_{j}-u_{j} \nabla u_{i}\right) /\left|u_{i} \nabla u_{j}-u_{j} \nabla u_{i}\right|$, where $u_{i}$ and $u_{j}$ are the $i$-th and $j$-th components of the vectorial order parameter $\mathbf{u} \in \mathbb{R}^{N}$.

In the vectorial case, the dependence of the limiting surface tensions and mobilities on the bulk potentials of the Allen-Cahn system is not given explicitly, but in terms of all the $N$ components of the planar stationary wave solutions. One of the issues of this paper is to find bulk potentials which allow a rather easy access to the resulting surface tensions and mobilities.

We compare numerical computations for planar and circular phase boundaries in two and three phase systems. The difference is, that in a three phase system, the third phase generally will be present in the interfacial region between two other phases. We demonstrate how this influences the solutions. In addition, we calculate the evolution of triple and quadruple junctions in three and four phase systems. Finally, we show a simulation of grain growth starting from many grains initially.
\end{abstract}

\section{INTRODUCTION}

Phase field models have been used to describe phase transition phenomena. In these models the phase boundary is modeled to be an interfacial layer of positive but small thickness. It has been shown that phase field concepts recover well known free boundary problems such as motion by mean curvature, the Stefan problem and the Mullins-Sekerka evolution, when the interfacial thickness tends to zero. In these sharp interface models, the transition takes place across a hypersurface. The relation between phase field models and sharp interface problems for the two phase case was first suggested by formal asymptotic expansions (see [25, 7]) and later proved rigorously (see [11, 30, 27] and the references therein). Bronsard and Reitich [5] were the first to study an Allen-Cahn system and they showed formally that in the sharp interface limit of the Allen-Cahn system one obtains an angle condition known as Young's law wherever three phases meet.

The aim of our work is to study the phase field concept for multi phase systems. We restrict ourselves to the theory of perfect conductors, i.e. temperature is constant, and the bulk phases influence the evolution of the interface only through a difference of constant bulk energies (see e.g. Angenent and Gurtin [2]). In this case, one has to study a system

1991 Mathematics Subject Classification. 35K55, 82C26, 65-05, 65M06, 35B25, 82C24.

Key words and phrases. phase field models, multi phase diffusion, Allen-Cahn systems, triple junction dynamics, numerical simulations. 
of parabolic equations of Allen-Cahn type. This system can also be used to describe the evolution of grain boundaries in multi phase polycrystalline materials. Hence, when using the term phases and phase fields we also think of grains and order parameters where the order parameters describe different orientational variants of the same phase. We hope this does not cause any confusion.

In a recent paper [15], we studied an Allen-Cahn system which is based on a GinzburgLandau free energy for a vector valued phase field $\mathbf{u} \in \mathbb{R}^{N}$

$$
\mathcal{E}(\mathbf{u})=\int_{\Omega}\left(\varepsilon f(\mathbf{u}, \nabla \mathbf{u})+\frac{1}{\varepsilon} \Psi(\mathbf{u})+\Phi(\mathbf{u})\right)(x) d x
$$

with a generalized gradient energy $f$ and bulk potentials $\Psi$ and $\Phi$. We demonstrated by formally matched asymptotic expansions that this Allen-Cahn system can be used to approximate the anisotropic multi phase curvature flow with driving forces given by differences in bulk energies. The new feature of the Ginzburg-Landau free energy we used is that the gradient part is based on antisymmetric terms which involve the phase field and its gradient. This goes back to the theory of irreducible representation (see Landau and Lifschitz [17]). To be more precise, we give $f$ in its isotropic version as

$$
f_{i s o}(\mathbf{u}, \nabla \mathbf{u})=\sum_{i, j=1, i<j}^{N} \frac{\tilde{\sigma}_{i j}}{\tilde{\mu}_{i j}}\left|u_{i} \nabla u_{j}-u_{j} \nabla u_{i}\right|^{2} .
$$

This type of free energy was first proposed for multi phase field models by Steinbach et al. [28]. For results which used phase field generalizations of the $\xi$-vector and the stress tensor to identify the asymptotic limit of multi phase systems we refer to Wheeler and McFadden [31] and Nestler and Wheeler [19]. Applications of a phase field model to describe solidification of a eutectic alloy was studied by Wheeler, McFadden and Boettinger [32] and for related results on Cahn-Hilliard systems see Bronsard, Garcke and Stoth [4] and Garcke and Novick-Cohen [16].

As a further extension of a model which is based on a free energy defined by (1) and (2), Nestler and Wheeler [21] introduced a free energy potential that also depends on concentration and temperature. The incorporation of bulk fields enables to treat phase transitions that appear in solidification processes of alloys by allowing different phases to have different compositions. The Gibbs free energies in the model of [21] ) are thermodynamically consistent and such that the properties and shapes of eutectic and peritectic phase diagrams are incorporated. Herewith, Nestler and Wheeler [21] developed a phase field model which describes realistic features of peritectic and eutectic solidification.

The aim of this paper is to perform numerical simulations to investigate the relation between the Allen-Cahn system and its asymptotic limit. To formulate this limit, it is necessary to determine several parameters such as the surface tensions and the mobilities which are associated to all possible pairs of phases. The surface tension between phases $i$ and $j$ is for any $\nu \in \mathbb{R}^{2}$ determined by the expression

$$
\sigma_{i j}(\nu)=2 \inf _{\mathbf{p}} \int_{-1}^{1} \sqrt{\Psi(\mathbf{p}) f\left(\mathbf{p}, \mathbf{p}^{\prime} \otimes \nu\right)} d y,
$$

where $\mathbf{p}$ ranges over all Lipschitz continuous functions $\mathbf{p}:[-1,1] \rightarrow \Sigma$, connecting the vectors $\mathbf{e}^{i}$ and $\mathbf{e}^{j}$. Here, $\Sigma=\left\{\mathbf{u} \in \mathbb{R}^{N}: \sum_{i=1}^{N} u_{i}=1\right\}$ and $\mathbf{e}^{i}$ denotes the $i$ th standard unit vector and $\Psi_{\mid \Sigma}$ is assumed to have global minima exactly at the $\mathbf{e}^{i}$. We associate the vector $\mathrm{e}^{i}$ with phase $i$ (see Section 2 for more details). One objective of this paper is 
to determine bulk potentials $\Psi$, which are good for phase field approximations of sharp interface problems.

First, we ask the free energy to have good calibration properties: In applications, the surface tensions are given and one wants to choose a gradient energy $f$ and a bulk potential $\Psi$ with enough degrees of freedom to allow to easily calibrate the free energy such that the given surface tensions are obtained by (3). We stress that this calibration problem does not appear for two phase systems, since there the explicit formula

$$
\sigma(\nu)=2 \int_{0}^{1} \sqrt{\Psi(p, 1-p) f((p, 1-p),(1,-1) \otimes \nu)} d p
$$

holds, if we impose that $f$ is homogeneous of degree 2 in the gradient variable. No such formula holds in the vectorial case.

The next requirement is the property of reduction to a two-phase system locally in interfacial regions: As we think of the component $u_{i}$ to be the fraction of phase $i$ we want a transition layer between phases $i$ and $j$ to have the property that $u_{k}=0$ for all $k \neq i, j$. It turns out that this is satisfied if the minimum in (3) has this property.

A third request, which is not as crucial as the other two and which we only want for computational convenience, is locality of the interfacial layer: While the standard smooth double well potential for the two phase case has an infinite transition layer, the double obstacle potential has a finite transition layer with the phase field being exactly \pm 1 away from a small interfacial layer which is of order $\varepsilon$ (see Blowey and Elliott [3] and Nochetto, Paolini and Verdi [23]). A similar property we require for the multi phase case.

To compare different potentials and to study how it is possible to calibrate parameters, we perform numerical simulations in planar and radial geometry with and without driving forces. It turns out that a modification of a multi obstacle potential has the best performance with respect to the three requirements stated above. Hence, we choose this potential for simulations in more complicated geometries. We simulate the evolution of triple junctions, the stability of quadruple points and perform a larger computation which demonstrates that it is possible to use the multi phase field method to simulate situations with four order parameters and lots of grains. We numerically verify the validity of qualitative features such as the well known von Neumann law. In this context, we also refer to the work of Chen and Yang [10] and Fan and Chen [14] who used a multiple order parameter model to perform numerical simulations for systems with many non conserved order parameters. Their main concern lies in the study of the long time behavior of such systems, and in particular they were interested in investigating laws for the evolution of the average domain size of different grain variants. In contrast to this, we concentrate on local qualitative features and especially we use the numerical simulations in order to relate order parameter models to sharp interface models. Other numerical methods, which have been used so far to simulate the evolution in multi phase systems, are based on sharp interface models. We refer to Bronsard and Wetton [6] and Neubauer [22] for numerical computations based on parameterizations of interfaces and to Merriman, Bence and Osher [18] for a level set approach for the motion of multiple junctions. So far, there appeared no simulations for an anisotropic multi phase system in the literature. In a forthcoming paper, we plan to present numerical simulations for the anisotropic multi phase field concept.

In Section 2, we introduce the multi phase field concept and state the corresponding sharp-interface model. We then give several forms of free energies and discuss how to 
calibrate the parameters (Section 3). In the main part of the paper (Section 4), we systematically compare results from numerical simulations to the predictions of the formal asymptotic expansion of [15]. In particular, we try to find a potential which makes it as easy as possible to calibrate the parameters. Finally, in an appendix, we present a self similar profile for the sharp interface model and show that this profile is exponentially attractive. We use this profile as a reference solution for our simulations.

\section{The Multi Phase Field CONCEPT}

In this section we introduce the multi phase field concept. We assume that $N$ phases are present in the system and we introduce a multi phase order parameter $\mathbf{u}$ which lies in $\mathbb{R}^{N}$. The $i$ th component $u_{i}$ of $\mathbf{u}$ stands for the fraction of phase $i$. Therefore, the phase space for a multi phase order parameter $\mathbf{u}$ is

$$
\Sigma:=\left\{\mathbf{u} \in \mathbb{R}^{N}: \sum_{i=1}^{N} u_{i}=1\right\} .
$$

In view of this constraint, it turns out to be convenient to introduce the projection $T$ onto the tangent plane $T \Sigma:=\left\{\mathbf{u} \in \mathbb{R}^{N}: \sum_{i=1}^{N} u_{i}=0\right\}$, by

$$
(T \mathbf{u})_{i}:=u_{i}-\frac{1}{N} \sum_{j=1}^{N} u_{j} .
$$

In addition, we ask all components of the order parameter $\mathbf{u}$ to be nonnegative, i.e., we want $\mathbf{u} \in G$, where

$$
G:=\left\{\mathbf{u} \in \Sigma: u_{i} \geq 0\right\}
$$

is the Gibbs simplex in $\mathbb{R}^{N}$. Now, let $\Omega$ be a bounded domain in $\mathbb{R}^{2}$ with outer normal $\nu_{\partial \Omega}$ and tangent $\tau_{\partial \Omega}$. Then, the vectorial Allen-Cahn system is the $L^{2}$-gradient flow of the free energy $\mathcal{E}($ see $(1))$ for a function

$$
\mathbf{u}:(0, T) \times \Omega \rightarrow \Sigma .
$$

The system takes the form

$$
\varepsilon \mathbf{u}_{t}=\varepsilon \operatorname{div} T f_{, X}(\mathbf{u}, \nabla \mathbf{u})-\varepsilon T f_{, \mathbf{u}}(\mathbf{u}, \nabla \mathbf{u})-\frac{1}{\varepsilon} T \Psi_{, \mathbf{u}}(\mathbf{u})-T \Phi_{, \mathbf{u}}(\mathbf{u}),
$$

together with the natural boundary condition

$$
T f_{, X}(\mathbf{u}, \nabla \mathbf{u}) \cdot \nu_{\partial \Omega}=0 .
$$

Here, $\varepsilon$ is a generally small length scale parameter. The functions $f, \Psi$ and $\Phi$ model the surface energy and the bulk energy, respectively, and we require:

$$
\begin{array}{ll}
f(\mathbf{u}, X) \geq 0, & \text { for } X=\left(X_{i j}\right)_{i=1, \ldots, N ; j=1, \ldots, n}, \\
\Psi(\mathbf{u}) \geq 0 & \text { and } \quad \Psi(\mathbf{u})=0 \Leftrightarrow \mathbf{u}=\mathbf{e}^{i},
\end{array}
$$

where $\mathbf{e}^{i}$ denotes the $i$-th standard unit vector in $\mathbb{R}^{N}$. In addition, we assume that $f$ is homogeneous of degree two and convex in the variable $X$. The function $\Phi$ models deviations from thermodynamical equilibrium.

If $N=2$ and $f=f_{\text {iso }}$ (see (2)), we recover the standard scalar Allen-Cahn equation for $\phi=u_{2}-u_{1}($ see $[15])$. 
As the parameter $\varepsilon$ gets small, the system (8), (9) aims to approximate a sharp interface model. In two spatial dimensions, the domain will be split into time dependent phases $\Omega_{i}$, where $\mathbf{u}$ takes the constant value $\mathbf{e}^{i}$. Those domains will be separated by interfacial curves $\Gamma$, and since we are dealing with a multi phase problem, those curves may intersect in triple (or even multiple) junctions $m$.

In its full generality, the governing equation for an interface $\Gamma$ separating bulk phases $\Omega_{i}$ and $\Omega_{j}$ is given by the kinetic Gibbs-Thomson law

$$
\hat{\mu}_{i j}(\theta) V=-\left(\hat{\sigma}_{i j}(\theta)+\hat{\sigma}_{i j}^{\prime \prime}(\theta)\right) \kappa+\Phi\left(\mathbf{e}^{j}\right)-\Phi\left(\mathbf{e}^{i}\right) .
$$

Here, $V$ and $\kappa$ are the normal velocity and the curvature of the interface $\Gamma$ with respect to the normal $\nu$, pointing from $\Omega_{i}$ to $\Omega_{j}$, and $\theta$ is the angle that the normal $\nu$ forms with the $x$-axis. (The sign of the curvature is chosen so, that the curvature of a ball with the normal pointing outwards is positive.) The coefficients $\hat{\mu}_{i j}$ and $\hat{\sigma}_{i j}$ are the mobility and the surface energy associated with a transition from phase $\Omega_{i}$ to $\Omega_{j}$. In the anisotropic case, both the mobility and the surface energy depend on the orientation of the interface.

At triple junctions $m$, where three interfaces $\Gamma_{1}, \Gamma_{2}$ and $\Gamma_{3}$, separating the bulk phases $\Omega_{i} \rightarrow \Omega_{j}, \Omega_{j} \rightarrow \Omega_{k}$ and $\Omega_{k} \rightarrow \Omega_{i}$, respectively, intersect, the force balance

$$
\left(\hat{\sigma}_{i j}\left(\theta_{1}\right) \tau_{1}-\hat{\sigma}_{i j}^{\prime}\left(\theta_{1}\right) \nu_{1}\right)+\left(\hat{\sigma}_{j k}\left(\theta_{2}\right) \tau_{2}-\hat{\sigma}_{j k}^{\prime}\left(\theta_{2}\right) \nu_{2}\right)+\left(\hat{\sigma}_{k i}\left(\theta_{3}\right) \tau_{3}-\hat{\sigma}_{k i}^{\prime}\left(\theta_{3}\right) \nu_{3}\right)=0
$$

holds. Here $\tau_{i}$ is the tangent to $\Gamma_{i}$, so that $\left(\nu_{i}, \tau_{i}\right)$ form a positively oriented basis of $\mathbb{R}^{2}$.

At boundary points $m \in \partial \Omega$, where an interface $\Gamma$, separating the bulk phases $\Omega_{i}$ and $\Omega_{j}$, intersects, the tangential force vanishes:

$$
\left(\hat{\sigma}_{i j}(\theta) \tau-\hat{\sigma}_{i j}^{\prime}(\theta) \nu\right) \cdot \tau_{\partial \Omega}=0 .
$$

For a derivation of this sharp interface model from the Allen-Cahn model we refer to previous work [15]. There we showed, that the surface energies $\hat{\sigma}_{i j}$ and $\hat{\mu}_{i j}$ associated with a transition from the $i$-th phase to the $j$-th phase can be determined from the energies $f$ and $\Psi$ of the Allen-Cahn model. The surface energy is given by

$$
\sigma_{i j}(\nu)=2 \inf _{\mathbf{p}} \int_{-1}^{1} \sqrt{\Psi(\mathbf{p}) f\left(\mathbf{p}, \mathbf{p}^{\prime} \otimes \nu\right)} d y,
$$

where $\mathbf{p}$ ranges over all Lipschitz continuous functions $\mathbf{p}:[-1,1] \rightarrow \Sigma$, connecting $\mathbf{e}^{i}$ to $\mathbf{e}^{j}$ (see also Sternberg [29]). The surface energy may as well be represented, using a solution $\mathbf{q}: \mathbb{R} \rightarrow \Sigma$ of the (reparameterized) Euler-Lagrange equation

$$
0=-\left(T f_{, X}\left(\mathbf{q}, \mathbf{q}^{\prime} \otimes \nu\right)\right)^{\prime} \nu+T f_{, \mathbf{u}}\left(\mathbf{q}, \mathbf{q}^{\prime} \otimes \nu\right)+T \Psi_{, \mathbf{u}}(\mathbf{q}),
$$

connecting $\mathbf{e}^{i}$ to $\mathbf{e}^{j}$. Then (for a suitable solution $\mathbf{q}$ of (14))

$$
\sigma_{i j}(\nu)=\int_{-\infty}^{\infty}\left(f\left(\mathbf{q}, \mathbf{q}^{\prime} \otimes \nu\right)+\Psi(\mathbf{q})\right) d z .
$$

The mobility $\mu_{i j}$ is given by

$$
\mu_{i j}(\nu)=\int_{-\infty}^{\infty}\left|\mathbf{q}^{\prime}\right|^{2} d z
$$

The quantities $\hat{\sigma}$ and $\hat{\mu}$ relate to $\sigma$ and $\mu$, respectively, via

$$
\hat{\sigma}(\theta)=\sigma(\nu(\theta)), \quad \hat{\mu}(\theta)=\mu(\nu(\theta)),
$$

with $\nu(\theta)=(\cos \theta, \sin \theta)$. 
In the present paper, we study the isotropic case. Hence, we associate to a transition from the $i$-phase to the $j$-phase a (constant) surface tension $\sigma_{i j}$ and a (constant) mobility $\mu_{i j}$ and to the $i$-phase we associate the bulk free energy $m_{i}$. The interface problem then consists of the kinetic Gibbs-Thomson law

$$
\mu_{i j} V=-\sigma_{i j} \kappa+m_{j}-m_{i}
$$

with the triple point condition

$$
\sigma_{i j} \tau_{1}+\sigma_{j k} \tau_{2}+\sigma_{k i} \tau_{3}=0
$$

and the boundary condition

$$
\tau \cdot \tau_{\partial \Omega}=0
$$

We note, that in order for the triple point condition to be solvable, we must always assume that

$$
\sigma_{i j}+\sigma_{j k}>\sigma_{k i}
$$

for all possible choices $i, j, k$. The triple point condition may as well be expressed in terms of the angles $\phi_{i}$ of the $i$-th phase (Young's law):

$$
\frac{\sin \phi_{i}}{\sigma_{j k}}=\frac{\sin \phi_{j}}{\sigma_{k i}}=\frac{\sin \phi_{k}}{\sigma_{i j}} \text {. }
$$

In practice, physical parameters like the surface tensions $\sigma_{i j}$, the mobilities $\mu_{i j}$ and the bulk free energies $m_{i}$ are given and one would like to choose the gradient energy $f$ and the potentials $\Psi$ and $\Phi$ such that the asymptotic limit of the Allen-Cahn system (8) satisfies (18)-(20) with the given parameters $\sigma_{i j}, \mu_{i j}$ and $m_{i}$. Comparing (10) and (18), we notice that we have to choose the potential $\Phi$ such that $\Phi\left(\mathbf{e}^{i}\right)=m_{i}$. The more difficult part is to determine the surface tensions and the mobilities in terms of $f$ and $\Psi$ out of the equations (15) and (16). Here, one needs to solve the minimum problem (13) which is in general difficult to solve explicitly in multi phase systems.

The question now is: How to choose the gradient energy $f$ and the potential $\Psi$ in order to obtain given surface energies and mobilities in the formulas (15) and (16) $\Gamma$ Our goal is to find $f$ and $\Psi$ such that it is easy to find the minimizer in (13), or at least to have a good approximative minimizer.

\section{The FREE ENERGIES}

In this section, we propose several forms of free energies and we discuss how it is possible to calibrate the parameters in the potential in order to recover given $\sigma$ 's and $\mu$ 's. We require a simple relation between the parameters in the functions $f$ and $\Psi$ and the given surface energies and mobilities. For $f$ we will always choose the isotropic surface potential (see [28], [15], [19])

$$
f_{\text {iso }}(\mathbf{u}, \nabla \mathbf{u}):=\sum_{i<j} \frac{\tilde{\sigma}_{i j}}{\tilde{\mu}_{i j}}\left|u_{i} \nabla u_{j}-u_{j} \nabla u_{i}\right|^{2}
$$


It is easily verified that this surface potential leads to isotropic surface energies and mobilities. As possible choices for $\Psi$, we either take the standard multi well bulk potential

$$
\Psi_{s t}(\mathbf{u}):=9 \sum_{i<j} \tilde{\mu}_{i j} \tilde{\sigma}_{i j} u_{i}^{2} u_{j}^{2},
$$

or a higher order variant

$$
\hat{\Psi}_{s t}(\mathbf{u}):=\Psi_{s t}(\mathbf{u})+\sum_{i<j<k} \sigma_{i j k} u_{i}^{2} u_{j}^{2} u_{k}^{2},
$$

or the multi obstacle potential (see [3], [13])

$$
\Psi_{o b}(\mathbf{u}):=\frac{16}{\pi^{2}} \sum_{i<j} \tilde{\mu}_{i j} \tilde{\sigma}_{i j} u_{i} u_{j},
$$

whenever $\mathbf{u} \in G$, and $\Psi_{o b}(\mathbf{u})=+\infty$, whenever $\mathbf{u} \notin G$, or a higher order variant

$$
\hat{\Psi}_{o b}(\mathbf{u}):=\Psi_{o b}(\mathbf{u})+\sum_{i<j<k} \sigma_{i j k} u_{i} u_{j} u_{k} .
$$

For the obstacle potentials the system of Allen-Cahn equations (8) has to be replaced by a parabolic variational inequality. We refer to Blowey and Elliott [3] for a precise statement of the formulation in the two phase case. The generalization of the variational inequality to $N$-phases is straightforward and we do not present the details here. The formal asymptotic expansions in [15] which related the multi phase field system to the sharp interface model only covers the case of the smooth potentials (24) and (25). But an analogous asymptotic is possible for the obstacle potentials (26) and (27).

For the lower order bulk free energy $\Phi$, we choose

$$
\Phi_{s t}(\mathbf{u}):=\sum_{i} m_{i} u_{i}^{2}\left(3-2 u_{i}\right)
$$

or

$$
\Phi_{o b}(\mathbf{u}):=\sum_{i} m_{i} u_{i}
$$

In two phase systems $(N=2)$ the standard bulk potential $\Psi_{s t}$ reduces to the standard quartic double well potential (see [15]) and the multi obstacle potential reduces to the double obstacle potential which was studied by Blowey and Elliott [3].

The parameters $\tilde{\mu}_{i j}$ and $\tilde{\sigma}_{i j}$ and the absolute numbers in the surface potential $f$ and the bulk potentials $\Psi$ are chosen with the following property. If, in (13) we only minimize over the smaller class of all $\mathbf{p}:[-1,1] \rightarrow \partial G$ connecting $\mathbf{e}^{i}$ to $\mathbf{e}^{j}$, then this minimum is exactly $\tilde{\sigma}_{i j}$, and the mobility calculated with the corresponding reparameterized minimizer $\mathbf{q}: \mathbb{R} \rightarrow \partial G$ is exactly $\tilde{\mu}_{i j}$. Thus, we will have $\sigma_{i j}=\tilde{\sigma}_{i j}$ and $\mu_{i j}=\tilde{\mu}_{i j}$, whenever the actual minimizer in (13) lies on the boundary of the Gibbs simplex. This is the case in the two phase problem $(N=2)$ with any of the potentials. In the case $N \geq 3$, the actual minimizer generally lies in the interior of the Gibbs simplex, and in the transition region connecting phase $i$ to phase $j$, the other $N-2$ phases are present. We then have $\sigma_{i j}<\tilde{\sigma}_{i j}$. The steeper the potential is in the interior of the Gibbs simplex, the better $\sigma_{i j} \approx \tilde{\sigma}_{i j}$.

As mentioned before, the parameters $\sigma_{i j}$ and $\mu_{i j}$ in practice are given physical parameters, and $\tilde{\sigma}_{i j}$ and $\tilde{\mu}_{i j}$ should be chosen so that the algorithm given by solving (14) and (15) or (16), respectively, gives $\sigma_{i j}$ and $\mu_{i j}$. Thus, it is important to have an easy access 
on the dependence of $\sigma_{i j}$ and $\mu_{i j}$ on $\tilde{\sigma}_{i j}$ and $\tilde{\mu}_{i j}$. In practical computations it turns out that for the higher order potentials with $\sigma_{i j k}$ chosen appropriately, it is sufficient to choose $\tilde{\sigma}_{i j}$ and $\tilde{\mu}_{i j}$ equal to $\sigma_{i j}$ and $\mu_{i j}$ because they fairly agree. For this reason, in most of our

calculations, we will either use $\hat{\Psi}_{s t}$ or $\hat{\Psi}_{o b}$. We will demonstrate the difference to choosing $\Psi_{s t}$ or $\Psi_{o b}$ in some experiments comparing the evolution of a 1-2-interface in a two phase system $(N=2)$ to the evolution of a $1-2$-interface in a three phase system $(N=3)$.

\section{Numerical Simulations}

We now describe the numerical method used to solve the initial value problem to (8) and (9). The domain $\Omega=(0, a) \times(0, b)$ is a rectangle, that we discretize uniformly with a cell size $\Delta x$. If not otherwise stated, we use $a=b=1.01, \varepsilon=0.1, \Delta x=\frac{1}{10} \varepsilon, \Delta t=\frac{1}{10}(\Delta x)^{2}$, where $\Delta t$ is the time step. We use an explicit algorithm to calculate the update for the new time step. We discretize all the differential operators, using nearest neighbors, and we implement the Neumann boundary condition by extending the values from the interior points constantly to the boundary. For the case of the obstacle potential, we first solve the smooth part of the differential equations, and then project back onto the Gibbs simplex.

In all the calculations, we always use the standard surface potential $f_{\text {iso }}$ given by (23). If not otherwise stated, we choose $\mu_{i j}=1$ and $\sigma_{i j k}=500$ for $\Psi=\hat{\Psi}_{s t}$, and $\sigma_{i j k}=5$ for $\Psi=\hat{\Psi}_{o b}$.

4.1. The CHOICE OF THE POTEnTIAL. In this section we investigate the dependence of $\sigma_{i j}$ and $\mu_{i j}$ on $\tilde{\sigma}_{i j}$ and $\tilde{\mu}_{i j}$. To this end, for a given set of parameters $\tilde{\sigma}_{i j}$ and $\tilde{\mu}_{i j}$, we calculate a solution of (14) for any of the potentials $\Psi_{s t}, \Psi_{o b}, \hat{\Psi}_{s t}$ and $\hat{\Psi}_{o b}$, by solving the time dependent problem with $\varepsilon=1$ and initial conditions making a transition from the corner $\mathbf{e}^{i}$ to the corner $\mathbf{e}^{j}$ of the Gibbs simplex. We compute until the solution gets numerically stationary. The final solution has one transition layer and is a numerical approximation of a solution to the ODE (14) connecting $\mathbf{e}^{i}$ to $\mathbf{e}^{j}$. We then use the result to determine $\sigma_{i j}$ and $\mu_{i j}$, using (15) and (16).

For the two phase case $(N=2)$, we choose the standard bulk potential $\Psi=\Psi_{s t}$ given by (24) with parameters $\tilde{\sigma}_{12}=\tilde{\mu}_{12}=1$. Then, numerically $\sigma_{12}=\mu_{12}=0.9969989$.

For the three phase case $(N=3)$, we choose any of the four potential $\Psi=\Psi_{s t}, \hat{\Psi}_{s t}$, $\Psi_{o b}, \hat{\Psi}_{o b}$, given by (24), (25), (26) and (27), with three choices of parameters

$$
\left(\tilde{\sigma}_{12}, \tilde{\sigma}_{23}, \tilde{\sigma}_{31}\right)=(1,1,1),(1,1,0.2),(1.8,1,1) .
$$

In Figure 1, we plot the solution to the ODE given by (14) for a 1-2 interface with $\left(\tilde{\sigma}_{12}, \tilde{\sigma}_{23}, \tilde{\sigma}_{31}\right)=(1,1,0.2)$. We observe that for $\Psi_{s t}, \hat{\Psi}_{s t}$ and $\Psi_{o b}$ the third phase appears in the transition layer. For all choices of the $\sigma$ 's stated above we observe that $\hat{\Psi}_{o b}$ has interfacial layers with $u_{3}$ being zero. The same is true for $\Psi_{o b}$, if all surface tensions are equal. But in general the solution $\mathbf{q}$ of (14) does not lie on the boundary of the Gibbs simplex.

For the potential $\hat{\Psi}_{s t}$, we noticed that the third component $u_{3}$ in an interfacial layer becomes smaller if we increase $\sigma_{i j k}$. In Figure 2, we plot the graph of the third component $q_{3}$ of the solution of the ODE for different values of $\sigma_{i j k}$. In addition, we give the graph of $\sigma_{12}$ as a function of $\sigma_{i j k}$, while all $\tilde{\sigma}_{i j}=1$. We see that $\sigma_{12}$ converges to $\tilde{\sigma}_{12}=1$ for large $\sigma_{i j k}$. 

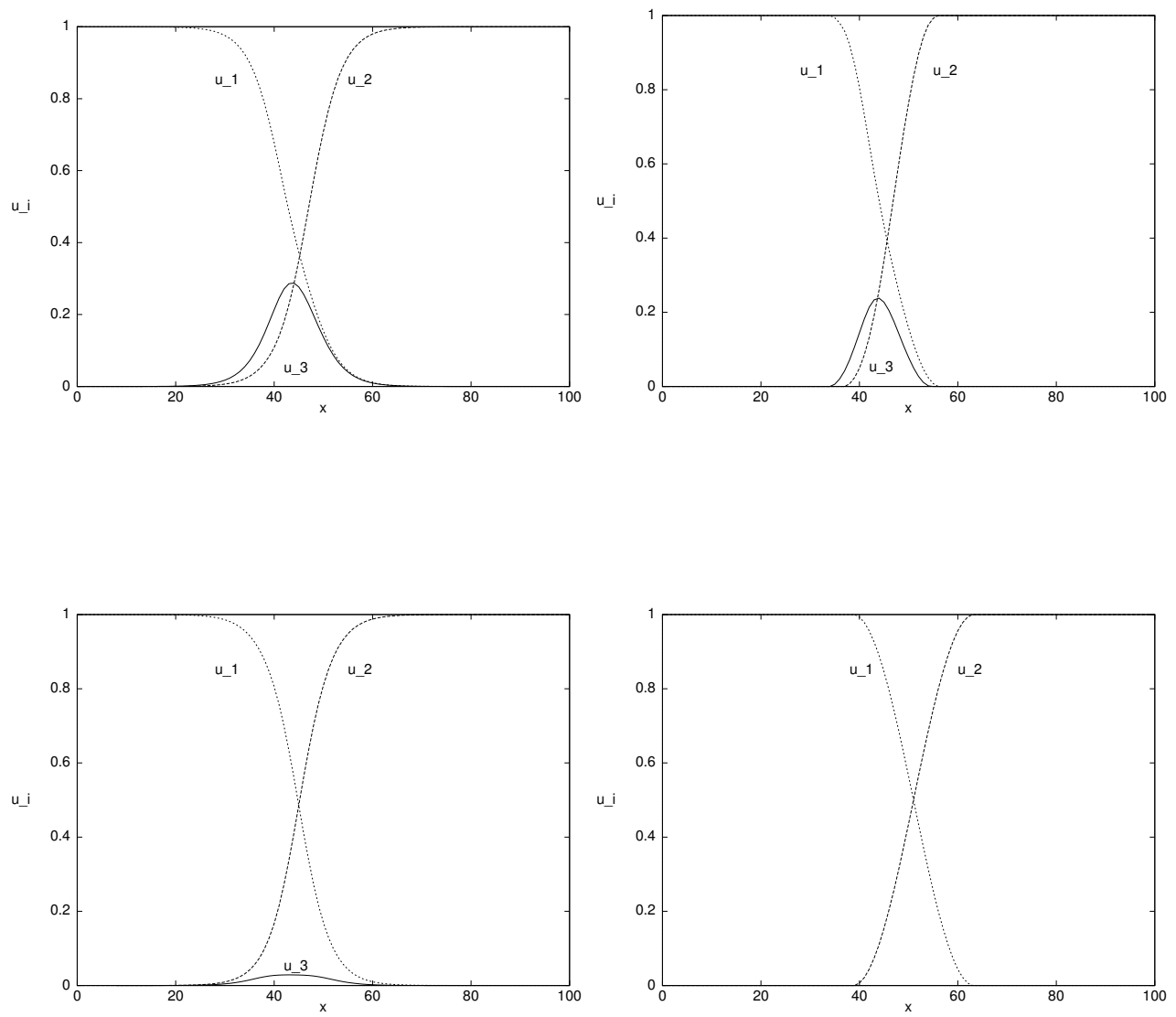

Figure 1. Plot of the components $u_{1}, u_{2}, u_{3}$ for the different choices of the potential $\Psi_{s t}, \Psi_{o b}, \hat{\Psi}_{s t}, \hat{\Psi}_{o b}$ (left to right and top to bottom) and with surface energy parameters $\tilde{\sigma}_{12}=\tilde{\sigma}_{23}=1 ; \tilde{\sigma}_{31}=0.2$.

In Table 1 , we give the values of $\sigma_{12}, \sigma_{23}, \sigma_{31}$ and $\mu_{12}, \mu_{23}, \mu_{31}$ and we observe that $\hat{\Psi}_{o b}$ gives the best results. This is meant in the sense that it is easy to calibrate the parameters in $\hat{\Psi}_{o b}$. The discrepancy between $\mu_{12}$ and $\tilde{\mu}_{12}$ and $\sigma_{12}$ and $\tilde{\sigma}_{12}$ is bigger for the standard or obstacle potential than for their higher order variants. This is due to the fact that for the higher order variant the connecting orbits are closer to the edges of the Gibbs simplex than for the potentials $\Psi_{s t}$ and $\Psi_{o b}$.

We thus conclude, that it is very difficult to properly calibrate the parameters in the potentials $\Psi_{s t}$ and $\Psi_{o b}$, by considering only phase transitions with the third phase not present. For the higher order variants of the potentials with a suitable big $\sigma_{i j k}$, we find that $\sigma \approx \tilde{\sigma}$ and $\mu \approx \tilde{\mu}$. But, in practice, we are not allowed to choose $\sigma_{i j k}$ arbitrarily large, since close to the center of the Gibbs simplex $\sigma_{i j k} u_{i}^{2} u_{j}^{2} u_{k}^{2} \approx \sigma_{i j k} 10^{-3}$ or $\sigma_{i j k} u_{i} u_{j} u_{k} \approx 4 \sigma_{i j k} 10^{-2}$ have to stay order of 1 with respect to $\varepsilon$. This motivates our choice of $\sigma_{i j k}=500$ or $\sigma_{i j k}=5$ for $\hat{\Psi}_{s t}$ or $\hat{\Psi}_{o b}$, respectively. 

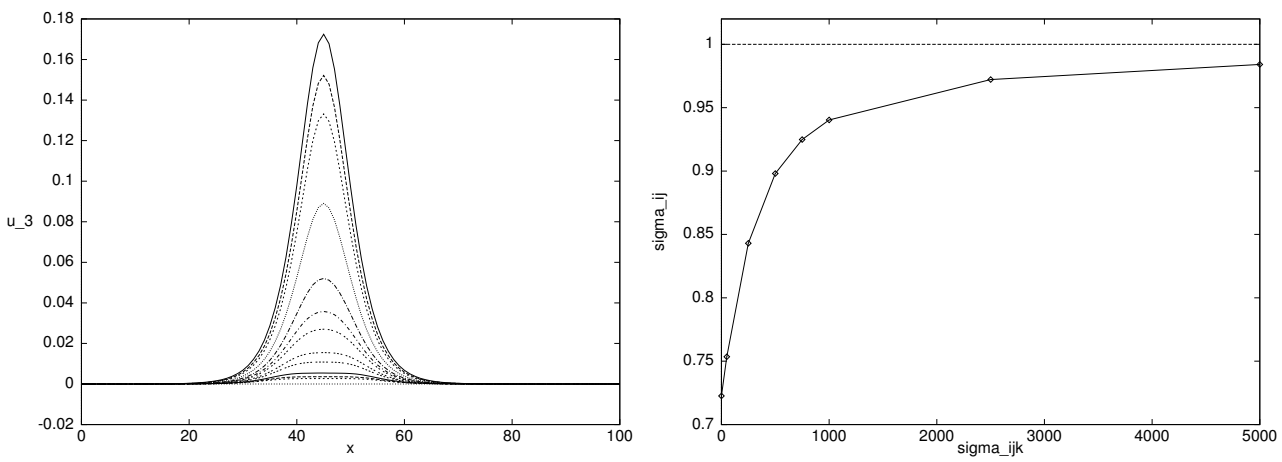

Figure 2. The first plot shows the third component of an 1-2 interfacial solution for the potential $\hat{\Psi}_{s t}$ using different values of $\sigma_{i j k}$. The second graph shows how good $\sigma_{12}$ approximates $\tilde{\sigma}_{12}$ as a function of $\sigma_{i j k}$

\begin{tabular}{|c|c|c|c|c|c|c|c|c|c|c|c|c|c|c|}
\hline $\begin{array}{l}\mu_{12} \\
\sigma_{12}\end{array}$ & $\begin{array}{l}\mu_{13} \\
\sigma_{13}\end{array}$ & $\begin{array}{l}\mu_{23} \\
\sigma_{23}\end{array}$ & \multicolumn{3}{|c|}{$\Psi_{s t}$} & \multicolumn{3}{|c|}{$\Psi_{a b}$} & \multicolumn{3}{|c|}{$\hat{\Psi}_{s t}$} & \multicolumn{3}{|c|}{$\hat{\Psi}_{o b}$} \\
\hline 1 & 1 & 1 & 1.008 & 1.008 & 1.008 & 0.997 & 0.997 & 0.997 & 1.014 & 1.014 & 1.014 & 0.996 & 0.996 & 0.996 \\
\hline 1 & 1 & 1 & 0.723 & 0.723 & 0.723 & 0.997 & 0.997 & 0.997 & 0.898 & 0.898 & 0.898 & 0.997 & 0.997 & 0.997 \\
\hline 1 & 1 & 1 & 0.988 & 1.056 & 1.056 & 0.997 & 1.367 & 1.367 & 0.998 & 1.019 & 1.019 & 0.996 & 0.997 & 0.997 \\
\hline 0.2 & 1 & 1 & 0.186 & 0.584 & 0.584 & 0.199 & 0.818 & 0.818 & 0.196 & 0.880 & 0.880 & 0.199 & 0.997 & 0.997 \\
\hline 1 & 1 & 1 & 0.992 & 1.074 & 0.992 & 0.997 & 1.377 & 0.997 & 1.009 & 1.065 & 1.009 & 0.996 & 0.996 & 0.996 \\
\hline 1 & 1.8 & 1 & 0.785 & 1.049 & 0.785 & 0.997 & 1.478 & 0.997 & 0.909 & 1.449 & 0.909 & 0.997 & 1.795 & 0.997 \\
\hline
\end{tabular}

TABLE 1. Calculated results of $\sigma_{i j}$ and $\mu_{i j}$ for given $\tilde{\sigma}_{i j}$ and $\tilde{\mu}_{i j}$, depending on the potential.

The higher order variant $\hat{\Psi}_{o b}$ of the obstacle potential $\Psi_{o b}$ proves to be very useful. It has the additional advantage, that the interfacial region is localized, so that in principle the phase field equations have only to be solved in some neighborhood of the interfaces.

4.2. Planar and circular interfaces in a two phase system $(N=2)$. For a two phase system $(N=2)$ we calculate the evolution of a planar interface under bulk driving forces, the evolution of a radial interface under the kinetic Gibbs-Thomson law with no bulk driving forces, and the evolution of a radial interface under the kinetic GibbsThomson law with bulk driving forces close to an equilibrium value.

Since for $N=2$ a calibration is not necessary we use the standard bulk potential $\Psi=\Psi_{s t}$ given by (24) with the parameter $\tilde{\sigma}_{12}=1$ and the forcing term $\Phi_{s t}$ given by (28). We describe the following three experiments. 
i) Planar interface with driving forces: As bulk energies, we choose $m_{2}=0$ and different values for $m_{1}$. In Table 2, we display $-V / m_{1}$, and observe a good agreement with the theoretical value 1 as given by the kinetic Gibbs-Thomson law (18).

\begin{tabular}{|l||c|c|c|c|c|c|c|c|c|c|c|c|}
\hline$m_{1}$ & -6 & -4 & -2 & -1 & 0 & 0.5 & 1 & 2 & 3 & 4 & 6 & 8 \\
\hline$\mu_{12}$ & 1.062 & 1 & 1 & 1 & 1 & 1 & 1 & 1 & 1.008 & 1.017 & 1.062 & 1.111 \\
\hline
\end{tabular}

TABLE 2. Calculated mobility $\mu_{12}$ for different bulk energies $m_{1}$.

ii) Radial interface without driving forces: We choose different initial configurations corresponding to radial interfaces with initial radius $r$ and we look at vanishing bulk driving forces $m_{1}=m_{2}=0$. In Figure 3 we plot the evolution of the radius as a function of time for different initial radii and compare the result to the theoretical values predicted by the kinetic Gibbs-Thomson law (18).

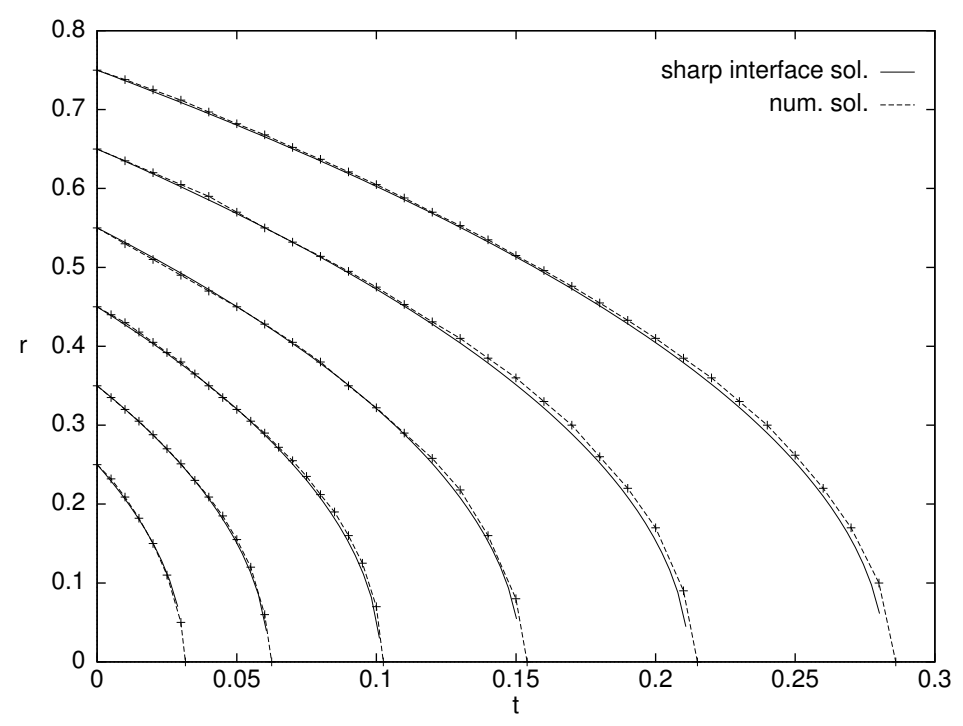

Figure 3. Plot of the dissolution process of spherical particles for different initial radii.

iii) Radial interface with driving forces close to equilibrium: We choose initial data with a spherical interface of radius $r=0.505$ with phase 1 lying inside the ball. As bulk energies we choose $m_{1}=0$ and vary $m_{2}$.

In Figure 4 we plot the evolution of the radius as a function of time for different values of the driving force $m:=m_{2}-m_{1}$. If $m$ is chosen so that $\frac{1}{r}=m$, then we observe that the evolution is stationary as predicted by the kinetic Gibbs-Thomson law (18), whereas in 


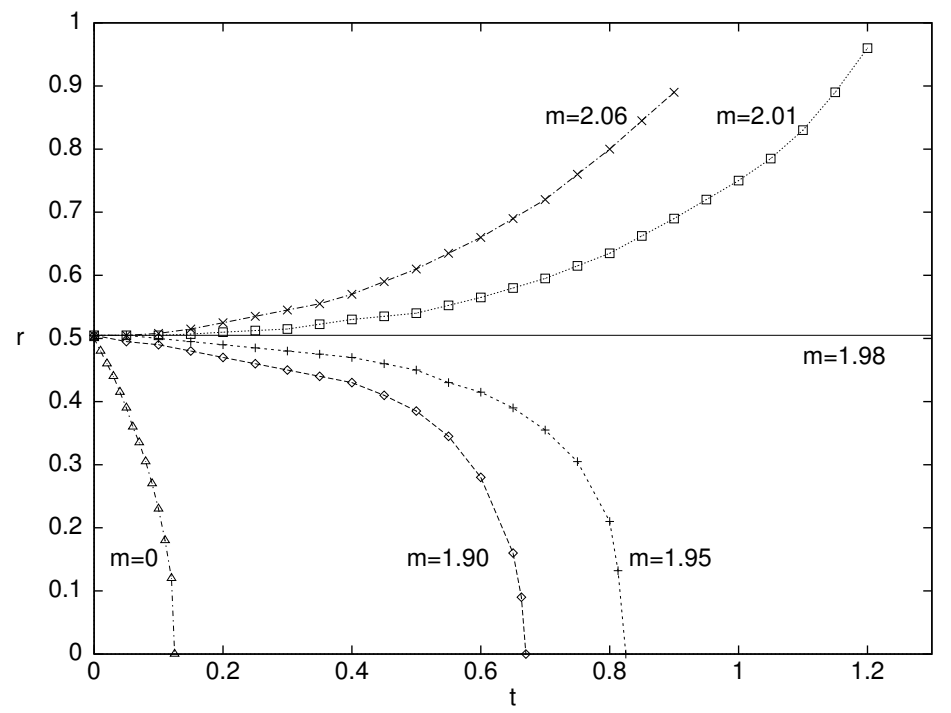

Figure 4. Calculated evolution of the radius as a function of time for different driving forces close to equilibrium.

the other cases the interface either shrinks or expands. This is due to the fact that $r=\frac{1}{m}$ is the critical radius for a nucleus, when the bulk energies are $m_{1}$ and $m_{2}$.

4.3. Two phase configurations in a three phase system $(N=3)$. For a three phase problem $(N=3)$, we calculate the evolution of planar and radial interfaces between the 1-phase and the 2-phase. We use

- $\Psi=\Psi_{s t}, \hat{\Psi}_{s t}$ given by (24), (25) with $\Phi=\Phi_{s t}$ given by (28),

- $\Psi=\Psi_{o b}, \hat{\Psi}_{o b}$ given by (26), (27) with $\Phi=\Phi_{o b}$ given by (29),

with parameters $\tilde{\sigma}_{12}=\tilde{\sigma}_{23}=\tilde{\sigma}_{31}=1$. We do the same experiments as in the two phase case $(N=2)$, always initially setting $u_{3} \equiv 0$. Our issue here is to study whether the third component of the phase field has an influence on a configuration which involves only two phases.

For the planar front experiment, we display $-V / m_{1}$ in Table 3 for the three different potentials. In all experiments $\tilde{\mu}_{12}=1$, and having in mind that our goal is to have $\tilde{\mu}_{12} \approx \mu_{12}=-V / m_{1}$, we see that all potentials give quite good results with the obstacle potentials being slightly better.

In Figure 5, we plot the evolution of the radius as a function of time for the radial interface experiment, using the four different potentials, and compare this to the solution of the sharp interface problem determined by the kinetic Gibbs-Thomson law $\mu_{12} \dot{r}=-\sigma_{12} \frac{1}{r}$ (18). The results for the obstacle potential $\Psi_{o b}$ and its higher order variant $\hat{\Psi}_{o b}$ are graphically indistinguishable. We stress, that the discrepancy in the results is not a numerical error, but due to the more or less successful calibration of the potentials as described in 


\begin{tabular}{|c||c|c|c|c|c|c|c|c|c|c|c|}
\hline$m_{1}$ & -4 & -2 & -1 & 0.5 & 1 & 2 & 3 & 4 & 6 & 8 & 12 \\
\hline$\Psi_{s t}$ & 0.966 & 0.982 & 1 & 1.014 & 1.015 & 1.029 & 1.053 & 1.048 & 1.059 & 1.096 & 1.086 \\
\hline$\Psi_{o b}$ & 1.109 & 1 & 1 & 1 & 1 & 1 & 1 & 0.992 & 1 & 0.983 & 0.940 \\
\hline$\hat{\Psi}_{s t}$ & 1.011 & 1.015 & 1 & 1 & 1 & 1.029 & 1.019 & 1.021 & 1.029 & 1.030 & 1.036 \\
\hline$\hat{\Psi}_{o b}$ & 0.989 & 1 & 1 & 1 & 1 & 1 & 1 & 0.985 & 0.985 & 0.971 & 0.913 \\
\hline
\end{tabular}

TABLE 3 . Calculated mobility $\mu_{12}$ depending on the driving force and the different potentials.

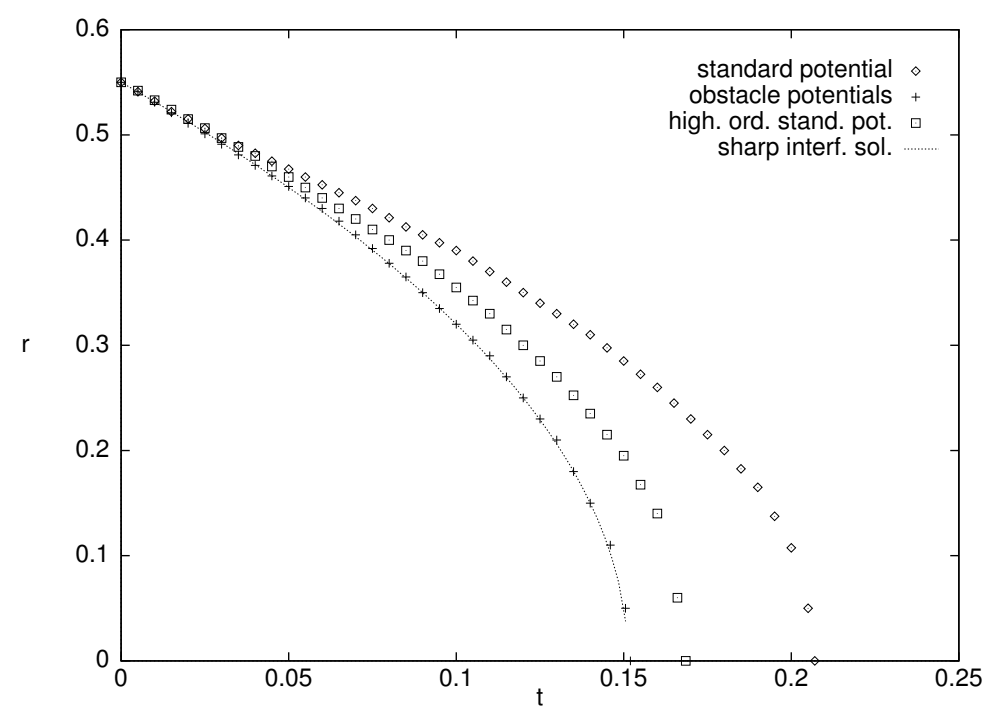

Figure 5. The radial interface experiment for different potentials

Paragraph 4.1. In this experiment, the two obstacle potentials clearly give the best results.

Finally, we give one result which is not covered by the asymptotic analysis of [15]. In Figure 6, we plot the graph of the first component $u_{1}$ of the solution for the obstacle potential $\Psi_{o b}$ with $\tilde{\sigma}_{i j}=\tilde{\mu}_{i j}=1$, with the parameters $\varepsilon=1, a=b=10.1$, and with driving forces $m_{2}=0$ and $m_{1}=\left(16 / \pi^{2}\right) \pm 0.0001$.

At the value $m_{1}=\left(16 / \pi^{2}\right)$ the potential part $(1 / \varepsilon) \Psi+\Phi$ of the free energy changes from being bistable into being stable, and only having one local minimum. For values slightly less than the critical value, we still observe a traveling wave solution connecting the two local minima, whereas for values slightly above the critical value, the traveling wave profile gets unstable. 

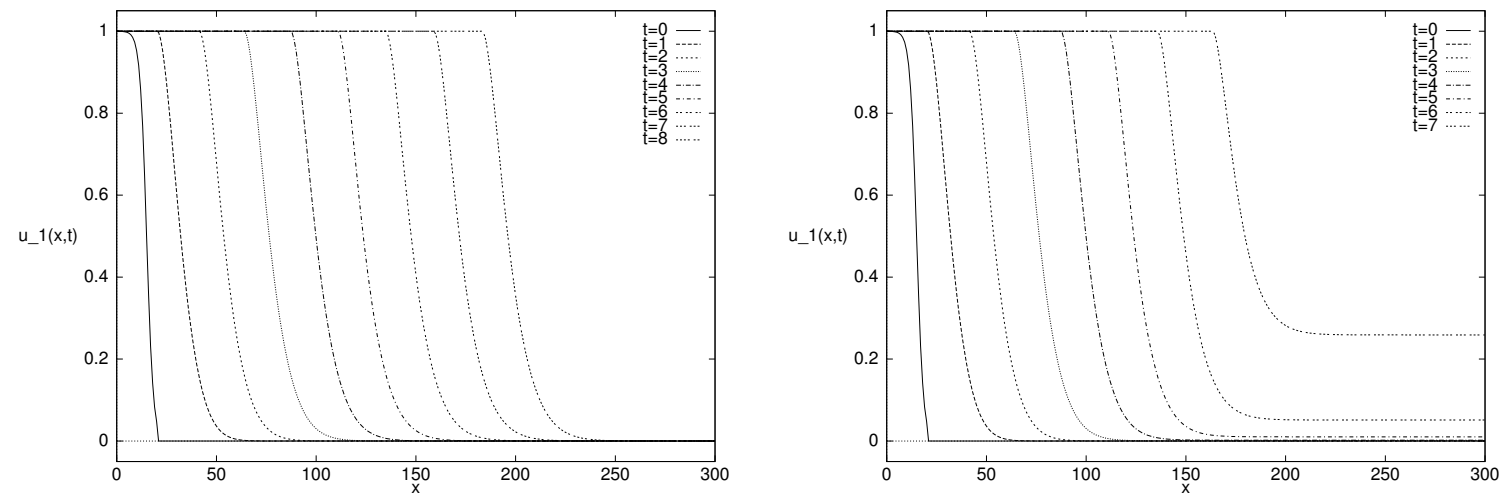

Figure 6. Transition from a stable (left) to an instable (right) profile of the component $u_{1}$ of the solution as the driving force becomes larger than $m_{1}=$ $\left(16 / \pi^{2}\right)$

4.4. Triple Junctions in a three phase system. For a three phase system $(N=3)$, we calculate the evolution of triple junctions. We choose $\varepsilon=0.05, \Psi=\hat{\Psi}_{o b}$ given by (27) with $\sigma_{i j k}=5$. If not otherwise stated, there will be no forcing terms $(\Phi \equiv 0)$.

For multi phase systems we determine the interfaces by the following strategy: We divide the Gibbs simplex into $\mathrm{N}$ parts, phase $i$ being represented by all $\mathbf{u}$ with $u_{i}$ bigger than the other components, and associate to any of the phases a color. Then, we divide the domain $\Omega$ into colored subregions according to the value of $\mathbf{u}$ in the numerical cells. Interfacial cells are then defined to be cells with a neighboring cell of different color. The location of the triple point is numerically determined by a maximum of $u_{1} \cdot u_{2} \cdot u_{3}$.

We do the following experiments:

i) Axially symmetric triple junction: We choose the parameters

$$
\tilde{\sigma}_{12}=\tilde{\sigma}_{13}=1 \quad \text { and } \quad \tilde{\sigma}_{23} \text { varies, }
$$

which correspond to the angle configurations

$$
\phi_{1} \text { varies and } \phi_{2}=\phi_{3} \text {. }
$$

As initial configuration, we choose a $\mathrm{T}$-shaped triple junction. We note, that this initial configuration is not consistent with Young's law (19).

In Figure 7, we plot the location of the interfaces at several times (for $\phi_{1}=90^{\circ}$ ), and we display an enlarged region around a triple junction. We note, that first very rapidly the angle condition is attained, and that then the shape of the interfaces starts to change, approaching a constantly transported profile. Finally, when the $2-3$ interface disappears the triple point vanishes and the 1-2 interface and the 3-1 interface shrink to the corners of the square. In an enlargement of the region around the triple junction we see that the $\phi_{1}=90^{\circ}$ angle is attained numerically.

For the sharp interface model (18), (19) and (20), the constantly transported profile can be constructed explicitly, and it turns out to be exponentially attractive. We give 

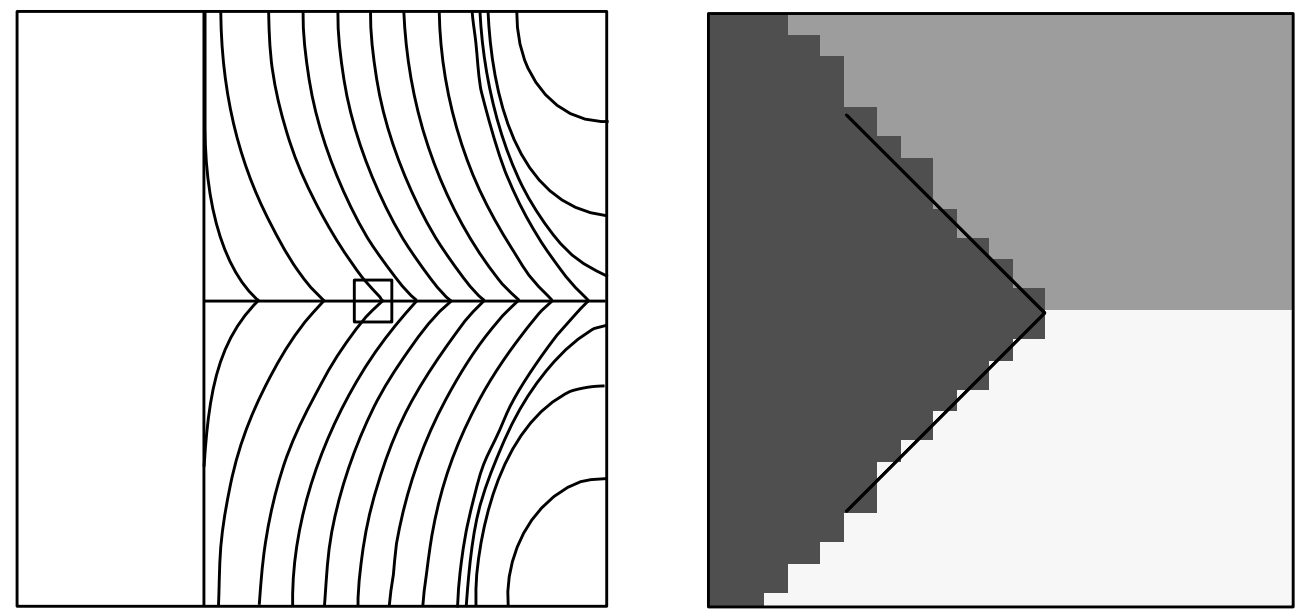

FIGURE 7. Simulated motion of the interfaces and the triple junction in a symmetric three phase configuration for different times.

the construction and a proof of this observation in the appendix. In Figure 8, we plot for $\phi_{1}=90^{\circ}$ the calculated transport velocities of the interface for different values on the $y$-axis and we see that they converge to the velocity of the explicit solution of the sharp interface problem.

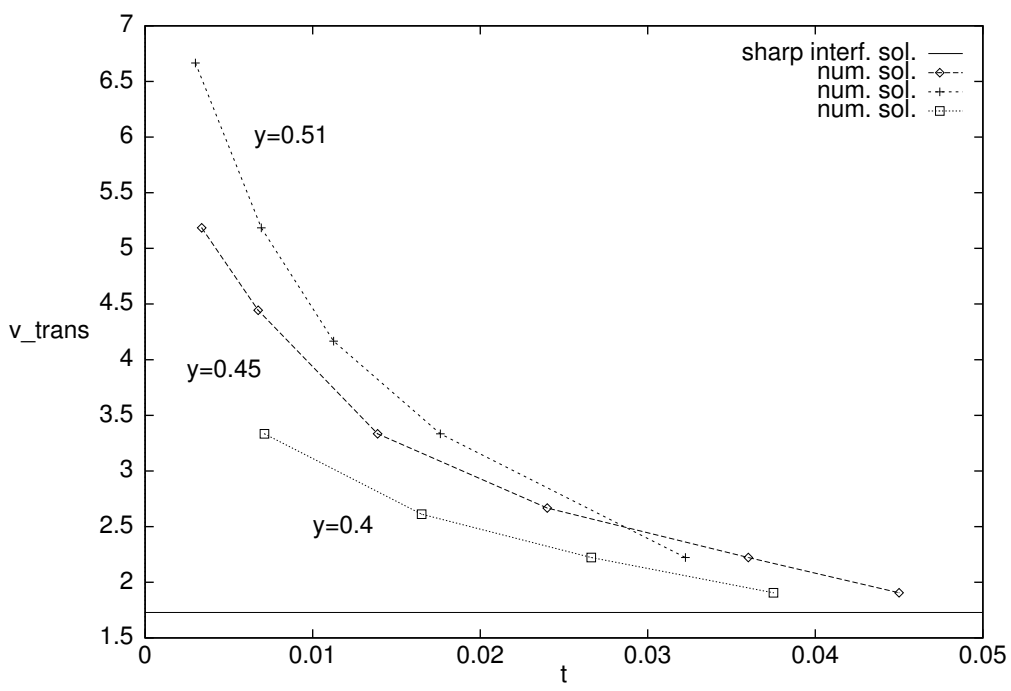

Figure 8. Transport velocities at different points on the $y$-axis compared to the velocity of the constantly transported solution of the sharp interface problem. 
In Figure 9, we compare the calculated solution to the profile of the explicit solution. In addition, we give the transport velocity $V_{\text {trans }}$ as a function of the angle $\phi_{1}$ and compare it to the theoretical value given in the appendix.
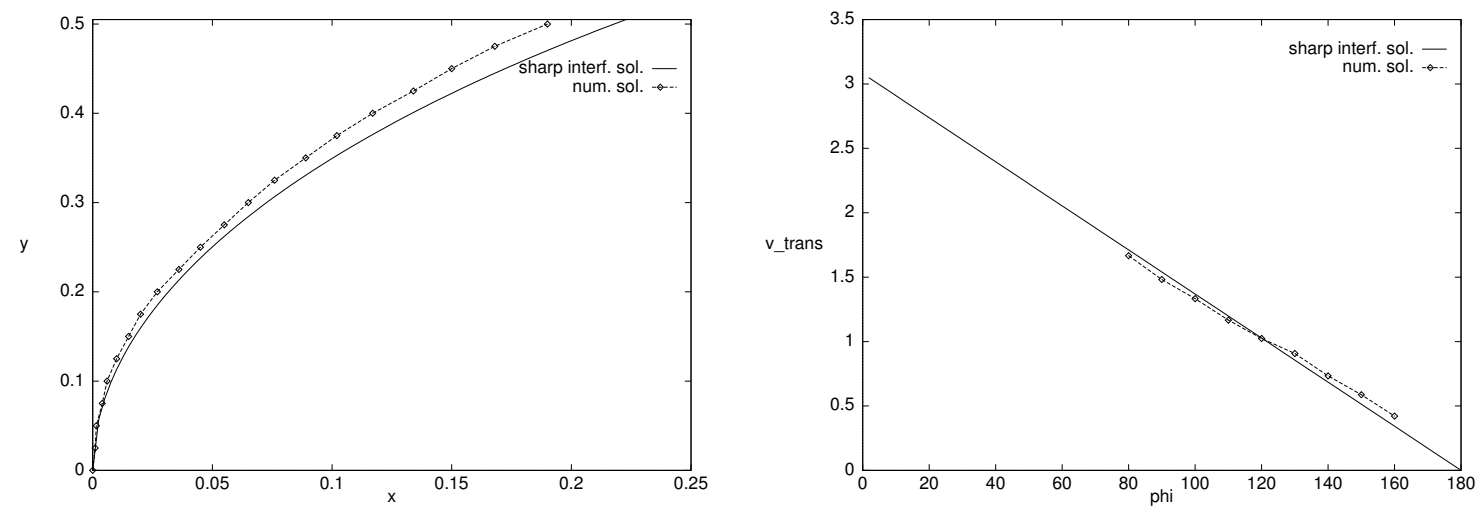

Figure 9. Comparison of the simulated interface profile (lower branch of Figure 7) with the constantly transported sharp interface solution (left). Calculated transport velocity as a function of the angle at the triple junction in comparison to the exact sharp interface solution (right).

We also performed numerical simulations with the constantly transported profile of the sharp interface problem as initial data. Here, we used different values of $\varepsilon$ and $\Delta x$ and we compared the error between the sharp interface solution and the numerical solution of the phase field system at later times. We found a high agreement and observed a reduction in the error as $\varepsilon$ and $\Delta x$ became smaller.

ii) Triple point evolution without axial symmetry: Now we choose

$$
\tilde{\sigma}_{12}=1 / \sqrt{3}, \quad \tilde{\sigma}_{13}=1, \quad \tilde{\sigma}_{23}=2 / \sqrt{3},
$$

which determines the angles of a triple junction to be

$$
\phi_{1}=90^{\circ}, \quad \phi_{2}=120^{\circ}, \quad \phi_{3}=150^{\circ} \text {. }
$$

As initial configuration we again choose a $\mathrm{T}$-shaped triple junction. In Figure 10, we plot the evolution of the triple point together with the location of the interfaces at different times. We observe that the angle condition at the triple junction are approximated rather good and that it is easy to determine the trace of the triple point.

iii) Triple point evolution under curvature and bulk driving forces: We choose two surface tensions the same, namely

$$
\tilde{\sigma}_{12}=1, \quad \tilde{\sigma}_{13}=1, \quad \tilde{\sigma}_{23}=\sqrt{2} .
$$

This choice of parameters corresponds to the angle configurations

$$
\phi_{1}=90^{\circ}, \quad \phi_{2}=135^{\circ}, \quad \phi_{3}=135^{\circ} .
$$




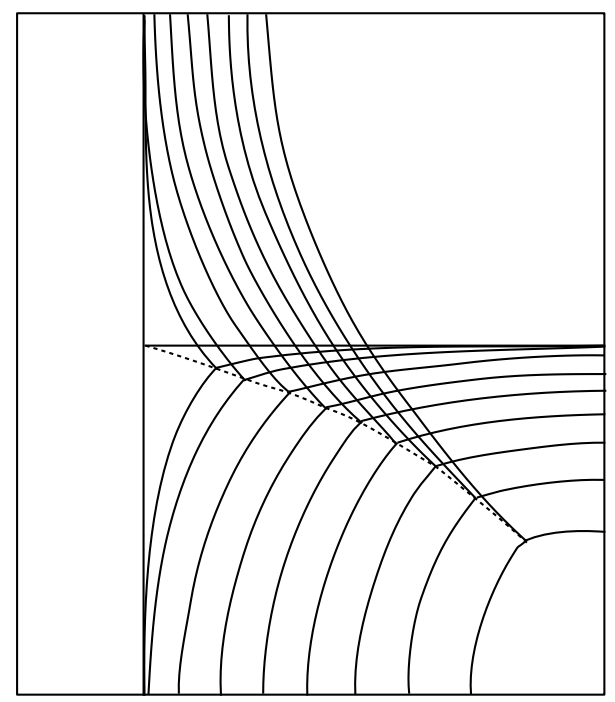

Figure 10. Numerical calculation of the triple junction evolution for the case of three different angles $\phi_{1}=90^{\circ}, \phi_{2}=120^{\circ}, \phi_{3}=150^{\circ}$.

We choose a nonzero driving force $\Phi=\Phi_{o b}$ given by (28) with parameters

$$
m_{1}=1, \quad m_{2}=2, \quad m_{3}=1 .
$$

As initial configuration we again choose a T-shaped triple junction. In Figure 11 we display the evolution of the interface at different times. Although the initial data are symmetric we have symmetry breaking because phase 2 (the phase to the top right) has a higher energy. The sharp interface problem (18)-(20) is a gradient flow of the energy

$$
\mathbf{E}:=\sum_{i, j} \sigma_{i j} \operatorname{Length}\left(\Gamma_{i j}\right)+\sum_{i} m_{i} \operatorname{Area}\left(\Omega_{i}\right)
$$

where $\Gamma_{i j}$ denotes an interface separating bulk phases $\Omega_{i}$ and $\Omega_{j}$ (see [24]). To decrease this energy it is efficient to decrease the area of phase 2 quickly. This effect is observed in our numerical simulations.

4.5. Stability of QuAdruple Junctions. For a system with four order parameters $(N=4)$, we choose numerical parameters as before, except that $\Delta t=\frac{1}{20}(\Delta x)^{2}$. We use the higher order obstacle potential $\Psi=\hat{\Psi}_{o b}$ given by $(27)$ with $\sigma_{i j k}=5$, no forcing term $(\Phi \equiv 0)$, parameters $\tilde{\sigma}_{12}=\tilde{\sigma}_{13}=\tilde{\sigma}_{24}=\tilde{\sigma}_{34}=\tilde{\sigma}:=1$ and we perform experiments for the following choices of the remaining surface tensions:

- $\tilde{\sigma}_{23}=\tilde{\sigma}_{14}=1$,

- $\tilde{\sigma}_{23}=\tilde{\sigma}_{14}=1.4$,

- $\tilde{\sigma}_{23}=\tilde{\sigma}_{14}=1.9$.

Initially we choose a cross shaped configuration, with the phases numbered from left to right and top to bottom. The values of $\mathbf{u}$ were chosen slightly perturbed in the 1-phase.

In Figure 12, we plot the evolution at different times for the three experiments. The location of the interfaces is plotted for the times $t=0,0.06,0.12,0.18, \ldots, 0.36$ in the first experiment and for the times $t=0,0.24,0.48,0.72,0.96$ in the second experiment. We calculated the third experiment until the time $t=2$ and the initial distribution of the phases remained the same. We see that the quadruple point is instable for the first two choices of 


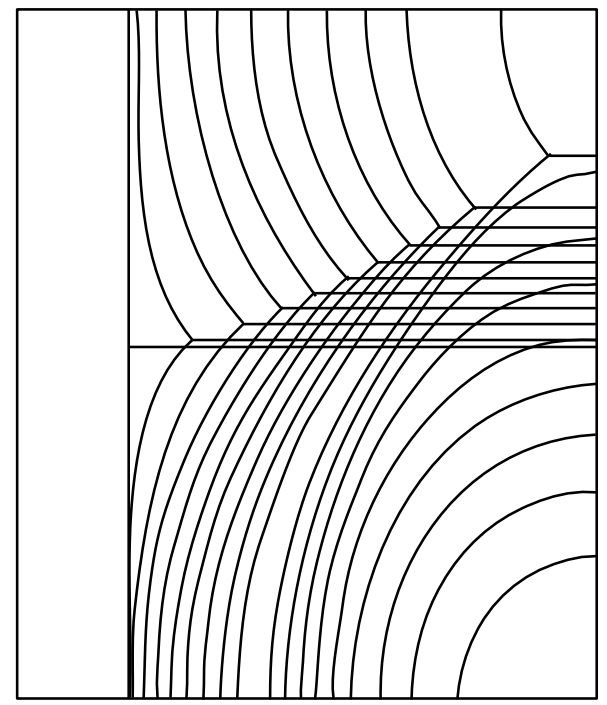

FiguRE 11. Triple junction motion driven by surface tension and bulk driving forces.

$\tilde{\sigma}_{23}$ and $\tilde{\sigma}_{14}$. In those cases, the quadruple point splits into two triple points and we observe that the evolution in the second case is much slower than for the first. This corresponds to the theoretical prediction that a quadruple point is instable if $\tilde{\sigma}_{23}, \tilde{\sigma}_{14}<\sqrt{2} \tilde{\sigma}$ and stable if $\tilde{\sigma}_{23}, \tilde{\sigma}_{14}>\sqrt{2} \tilde{\sigma}$. This was noticed by Cahn, Holm and Srolovitz [8]. Graphically, their condition can be interpreted as follows: A quadruple point splits into two triple junctions if the two outer angles in the triple junctions are larger than the corresponding angles in the quadruple junction (for a precise statement see [8]).
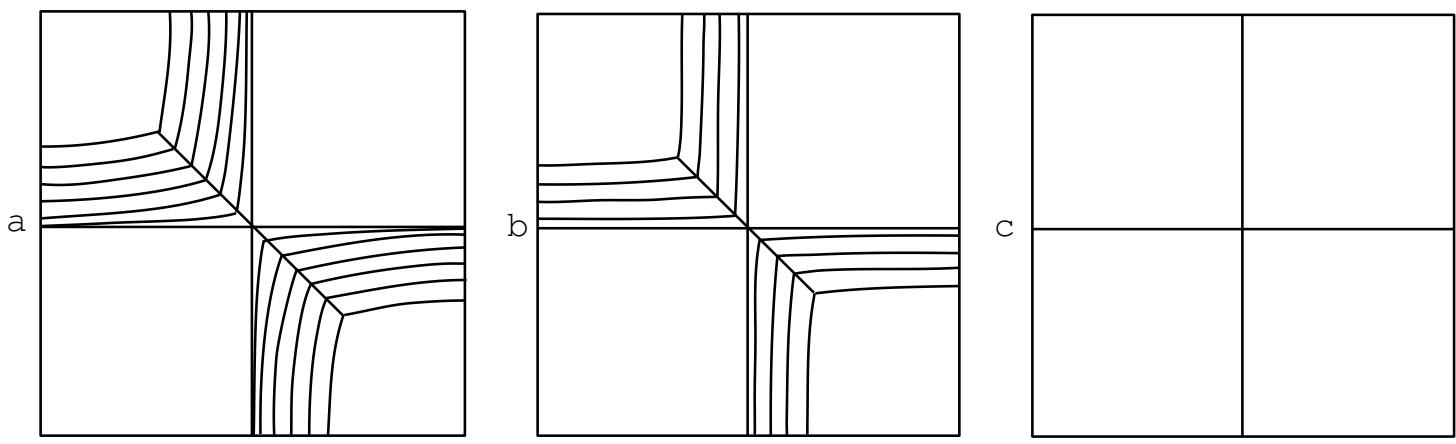

Figure 12. Numerical calculation with a quadruple junction as initial condition for different surface tensions.

4.6. Application to Grain Growth. Finally, we performed a larger simulation to demonstrate that the multi phase field model can be used to describe grain growth evolution. In this context, one should rather speak of a multi order parameter model because the components of $\mathbf{u}$ describe different oriententional variants in a crystalline material. In such systems, $120^{\circ}$ degree angle conditions are observed and therefore, we choose all surface tensions to be equal, in particular we set them equal to 1 . The calculations shown in Figure 13 were performed for $N=4, \varepsilon=0.02, \Delta x=0.0033$ and with the higher order obstacle potential $\Psi=\hat{\Psi}_{o b}$ given by $(27)$ with $\sigma_{i j k}=5$. 

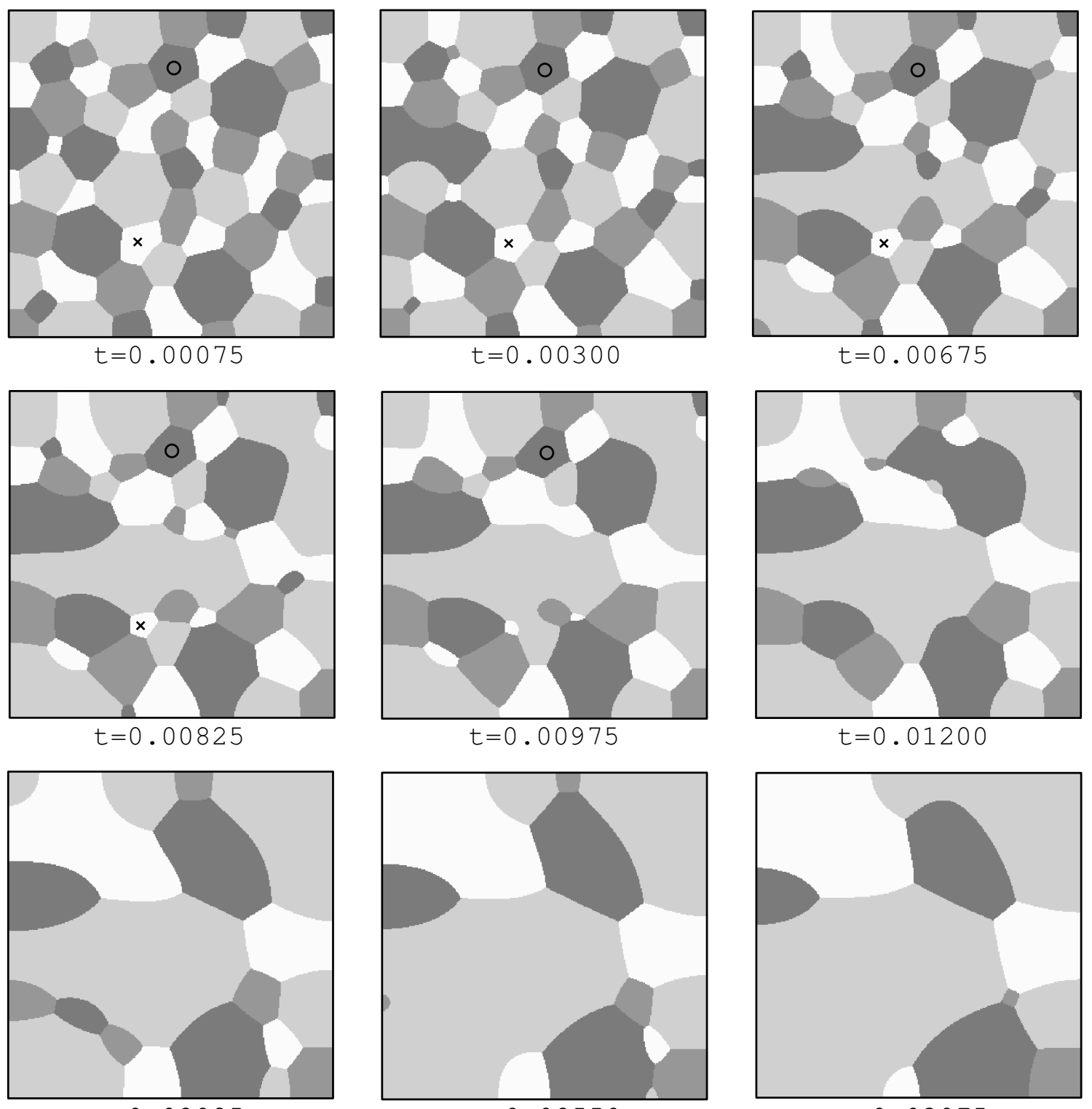

$t=0.02025$

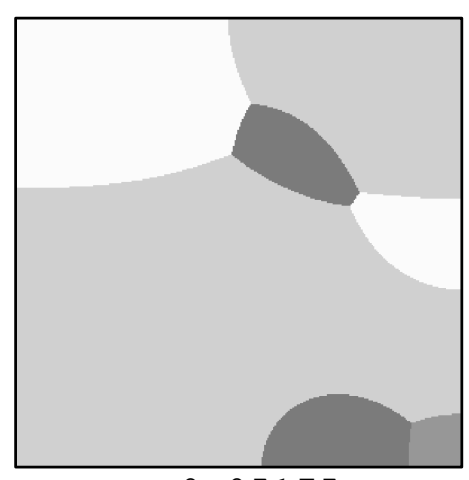

$t=0.05175$

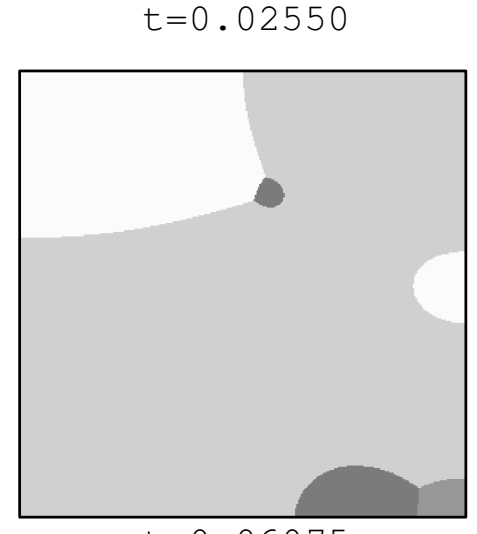

$t=0.03075$

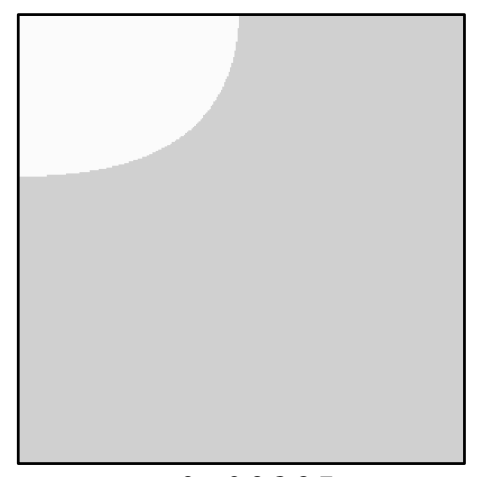

$t=0.08325$

Figure 13. Simulation of grain growth 
The von Neumann law says, that if $n$ curves enclose a region $D(t)$ at time $t$, and any of the curves moves by curvature $V=\kappa$, keeping a $120^{\circ}$ angle condition at intersections, then

$$
\frac{d}{d t} \operatorname{Area}(D(t))=\frac{\pi}{3}(n-6)
$$

In application to grain growth, this means that grains with less than six neighbors shrink, grains with more than six neighbors grow and six-sided grains keep their area in time. In Figure 13, we marked by a cross a region which shrinks and by circle a region which keeps the area. We remark that the number of neighbors of a grain changes in time and therefore the value of $n$ in (30) is constant only for a certain period of time.

\section{Conclusions}

We performed numerical simulations for a multi phase field model and compared the results to a sharp interface model where the evolution of the interface is governed by the kinetic Gibbs-Thomson law. In two phase configurations we found a high agreement between the two models even when the interfacial thickness was rather large (see Paragraphs 4.2 and 4.3 ). In most of our computations it was sufficient to choose the parameter $\varepsilon$ which is proportional to the interfacial thickness equal to $1 / 10$ or $1 / 20$. For three phase systems including triple junctions our numerical simulations support the formal asymptotic expansions of [15]. This is true for triple junctions evolving under curvature and bulk driving forces as well as for four phase systems including quadruple junctions and grain growth simulations. But we point out that the numerical parameters have to be chosen carefully. For example, if the parameters $\sigma_{i j k}$ in the higher order variants of the potentials are too large we observed qualitatively wrong results for the evolution of the triple junctions. For a three phase system one has to choose $(1 / 3)^{3} \sigma_{i j k}$ of order 1 to get results which agree with the formal asymptotic expansions of [15] (see Paragraph 4.1).

We also performed numerical simulations with mobilities $\mu_{i j}$ that are not equal. Here, we observed that the discretization has to be chosen carefully. If we first calculate the divergence in equation ( 8 ) by hand and then discretize the resulting terms, we noticed that the angle conditions were not resolved correctly in the numerical simulations. Whereas, if we discretize the gradient by forward differences and the divergence by backward differences we get a stable scheme which gives results in agreement with the asymptotic analysis. It seems that the first discretisation destroys too much of the structure of the problem, leading to a scheme which is less robust.

One of our main goals was to compare different potentials $\Psi$. It turns out that a higher order variant of the multi obstacle potential has the best properties. This means it fulfills the three requirements stated in the introduction: It is easy to adjust the parameters in the potential to given physical data (good calibration property), in an $i-j$ transition layer only the order parameters $i$ and $j$ are present (reduction to a two-phase system locally) and the interfacial region has a finite width (locality of the interfacial layer).

In the case of the higher order obstacle potential, the explicit algorithm can be made more efficient by using the property of locality of the interfaces. It is only necessary to compute the solution in a small discrete transition layer. For the two phase case, this fact has been exploited by the dynamic mesh algorithm (DMA) of Nochetto. Paolini and Verdi [23] and by the "mask" method of Elliott and Gardiner [12], and a generalization to multi phase systems is possible. Let us point out that spectral methods which have been used 
widely for numerical simulation of phase field type equations (see [9]) can not be used for our phase field system. This is due to the fact that our equation is not in a semilinear form. Hence, we believe that an implementation of an algorithm which uses the locality of the interfaces will be the most efficient one.

\section{APPENDIX}

Here, we give the derivation of a constantly transported profile in the axially symmetric case $\sigma_{12}=\sigma_{13}$ and $\mu_{12}=\mu_{13}$. We assume, that $\Omega=(0, a) \times \mathbb{R}$, the interface between the $2-$ phase and the $3-$ phase is located on the line $x=a / 2$ and that the interface between the 1 -phase and the 2-phase is a graph $x \mapsto y=u(t, x)$ for $x \in(0, a / 2)$, whereas the interface between the 1-phase and the 3 -phase is the graph $x \mapsto y=u(t, a-x)$ for $x \in(a / 2, a)$. Then a constantly transported profile takes the form

$$
u(t, x)=v(x)+c t .
$$

The differential equation (18) implies that

$$
\mu_{12} c=\sigma_{12} \frac{v^{\prime \prime}}{1+\left(v^{\prime}\right)^{2}}=\sigma_{12}\left(\arctan v^{\prime}\right)^{\prime}
$$

The Neumann condition $(20)$ gives $v^{\prime}(0)=0$, and thus

$$
\frac{\mu_{12}}{\sigma_{12}} c x=\arctan v^{\prime}(x) \text {. }
$$

Young's law (19) at the triple junction gives $v^{\prime}(a / 2)=\cot \left(\phi_{1} / 2\right)$, where $\phi_{1}$ is given by $\cos \left(\phi_{1} / 2\right)=\sigma_{23} /\left(2 \sigma_{12}\right)$. Thus, we find for the transport velocity $c$

$$
\frac{a \mu_{12}}{2 \sigma_{12}} c=\frac{\pi}{2}-\frac{\phi_{1}}{2}=\arctan \sqrt{\frac{\sigma_{23}^{2}}{4 \sigma_{12}^{2}-\sigma_{23}^{2}}},
$$

and the shape of the profile may be determined using

$$
v(x)=-\frac{\sigma_{12}}{\mu_{12} c} \log \cos \left(\frac{\mu_{12} c}{\sigma_{12}} x\right)+d .
$$

The constant of integration may be chosen appropriately. Now, we show that the constantly transported profile is exponentially attractive. We assume that $\tilde{u}$ were an arbitrary smooth graph, satisfying the kinetic Gibbs-Thomson law (w.l.o.g. we assume $\mu_{12}=\sigma_{12}$ )

$$
\tilde{u}_{t}=\frac{\tilde{u}^{\prime \prime}}{1+\left(\tilde{u}^{\prime}\right)^{2}}
$$

with

$$
\tilde{u}^{\prime}(0)=0, \quad \tilde{u}^{\prime}(a / 2)=\cot \left(\phi_{1} / 2\right)
$$

We take $u(t, x)=v(x)+c t$, where $v$ is the above constructed function with the constant of integration chosen such that

$$
\int_{0}^{a / 2}(\tilde{u}(0, x)-v(x)) d x=0 .
$$


Then, since $u(t, x)=v(x)+c t$ solves the same differential equation as $\tilde{u}$, we obtain for $w:=\tilde{u}-u$

$w_{t}=\frac{\tilde{u}^{\prime \prime}}{1+\left(\tilde{u}^{\prime}\right)^{2}}-\frac{u^{\prime \prime}}{1+\left(u^{\prime}\right)^{2}}=\left(\arctan \tilde{u}^{\prime}-\arctan u^{\prime}\right)^{\prime}, \quad \int_{0}^{a / 2} w(t, x) d x=0, \quad w_{\mid x=0, a / 2}^{\prime}=0$.

Multiplying this by $w$ and integrating the resulting identity results in

$$
\frac{1}{2} \frac{d}{d t} \int_{0}^{a / 2} w^{2}=-\int_{0}^{a / 2}\left(\arctan \tilde{u}^{\prime}-\arctan u^{\prime}\right)\left(u^{\prime}-v^{\prime}\right) \leq-C \int_{0}^{a / 2}\left(w^{\prime}\right)^{2} \leq-C \int_{0}^{a / 2} w^{2} .
$$

In the first inequality, we used the boundedness of $\tilde{u}^{\prime}$ (which follows from the maximum principle) and the monotonicity of the arctan, whereas in the second inequality, we used Poincaré's inequality for functions of mean value 0. Now, Gronwall's inequality finally implies the exponential decay of $\int_{0}^{a / 2} w^{2}$.

Acknowledgments: The first author thanks James F. Blowey for helpful discussions. This work was partially supported by the German Research Foundation (DFG) through the SFB256 Nichtlineare Partielle Differentialgleichungen and the grant SA335/25-2 Kurzzeitmetallurgie.

\section{REFERENCES}

[1] S. M. Allen and J. W. Cahn, A microscopic theory for antiphase motion and its application to antiphase domain coarsening, Acta Metall. Mater. 27 (1979), pp. 1085-1095.

[2] S. Angenent and M. E. Gurtin, Multi phase thermomechanics with interfacial structure 2. Evolution of an isothermal interface, Arch. Rat. Mech. Anal. 108 (1989), pp. 323-391.

[3] J. F. Blowey and C. M. Elliott, Curvature dependent phase boundary motion and parabolic obstacle problems, Proceedings of the IMA Workshop held at the University of Minnesota, Minneapolis, Minnesota, May 13-18, 1991, edited by Wei-Ming Ni, L. A. Peletier and J. L. Vazquez, IMA Volumes in Mathematics and its Applications, 47 (1993), Springer-Verlag, New York, pp. $19-60$.

[4] L. Bronsard, H. Garcke and B. Stoth, A multi-phase Mullins-Sekerka system: matched asymptotic expansions and an implicit time discretization for the geometric evolution problem, Proc. Roy. Soc. Edinb. 128 A, (1998) (to appear).

[5] L. Bronsard and F. Reitich, On three-phase boundary motion and the singular limit of a vectorvalued Ginzburg-Landau equation, Arch. Rat. Mech. Anal. 124 (1993), pp. 355-379.

[6] L. Bronsard and B. T. R. Wetton, A numerical method for tracking curve networks moving with curvature motion, J. of Computat. Phys. 120 (1995), pp. 66-87.

[7] G. Caginalp, Stefan and Hele Shaw type models as asymptotic limits of the phase field equations, Phys. Rev. A 39 No. 11 (1989), pp. 5887-5896.

[8] J. W. Cahn, E. A Holm and D. J. Srolovitz, Modeling microstructural evolution in two dimensional two-phase microstructures, Mat. Sci. For. Vols. 94-96 (1992), pp. 141-158.

[9] L. Q. Chen and J. Shen, Applications of semi-implicit Fourier spectral method to phase field equations, Computer Physics Communications 108 (1998), pp. 147-158.

[10] L. Q. Chen and W. Yang, Computer simulation of the domain dynamics of a quenched system with a large number of nonconserved order parameters: the grain-growth kinetics, Phys. Rev. B 50 No. 21 (1994), pp. 15752-15756.

[11] P. De Mottoni and M. Schatzman, Geometrical evolution of developed interfaces, Trans. AMS 347 (1995), pp. 1533-1589.

[12] C. M. Elliott and A. R. Gardiner, Double obstacle phase field computations of dendritic growth, University of Sussex CMAIA Research report 96-17.

[13] C. M. Elliott and St. Luckhaus, A generalized diffusion equation for phase separation of a multicomponent mixture with interfacial free energy, SFB256 University Bonn, Preprint No. 195 (1991). 
[14] D. N. Fan and L. Q. Chen, Diffuse-Interface Description of Grain Boundary Motion, Philosophical Magazine Letters 75 (1997), pp. 187-196.

[15] H. Garcke, B. Nestler and B. Stoth, On anisotropic order parameter models for multi-phase systems and their sharp interface limits, Physica D, to appear.

[16] H. Garcke and A. Novick-Cohen, A singular limit for a system of degenerate Cahn-Hilliard equations, SFB256 University Bonn, Preprint No. 505 (1997) (submitted).

[17] L. D. Landau and E. M. Lifschitz, Lehrbuch der theoretischen Physik, Band 5, Statistische Physik Teil I, Akademie Verlag Berlin (1979).

[18] B. Merriman, J. K. Bence and S. J. Osher, Motion of multiple junctions: a level set approach, J. Comput. Phys. 112 No. 2 (1994), pp. 334-363.

[19] B. Nestler and A. A. Wheeler, An anisotropic multi-phase-field model: interfaces and junctions, to appear in Phys. Rev. E.

[20] J. von Neumann, discussion to reference [26] in Metal Interfaces (ASM, Cleveland, OH, 1952) p. 108.

[21] B. Nestler amd A. A. Wheeler, On a multiphase-field model for solidification of peritectic and eutectic alloys, in preparation.

[22] R. Neubauer, diploma thesis, University Bonn (in preparation).

[23] R. H. Nochetto, M. Paolini and C. Verdi, A dynamic mesh method for curvature dependent evolving interfaces, J. Comput. Phys. 123 (1996), pp. 296-310.

[24] F. Reitich and H. M. Soner, Three-phase boundary motions under constant velocities. I: The vanishing surface tension limit, Proc. Roy. Soc. Edinb. A 126 (1996), pp. 837-865.

[25] J. Rubinstein, P. Sternberg and J. B. Keller, Fast reaction, slow diffusion and curve shortening, SIAM J. Appl. Math. 49 (1989), pp. 116-133.

[26] C. S. Smith, Grain shapes and other metallurgical applications of topology, in Metal Interfaces(ASM, Cleveland, OH, 1952) p. 65.

[27] H.M. Soner, Convergence of the phase-field equations to the Mullins-Sekerka problem with kinetic undercooling, Arch. Rat. Mech. Anal. 131 (1995), pp. 139-197.

[28] I. Steinbach, F. Pezolla, B. Nestler, M. Seeßelberg, R. Prieler, G. J. Schmitz and J. L. L. Rezende, A phase field concept for multi phase systems, Physica D 94 (1996), pp. 35-147.

[29] P. Sternberg, Vector-valued local minimizers of nonconvex variational problems, Rocky Mt. J. Math. 21 No. 2 (1991), pp. 799-807.

[30] B. Stoth, A sharp interface limit of the phase field equations, one-dimensional and axisymmetric, Europ. J. Appl. Math. 7 (1996), pp. 603-633.

[31] A. A. Wheeler and G. B. McFadden, On the notion of a $\xi$-vector and a stress tensor for a general class of anisotropic diffuse interface models, Proc. Roy. Soc. Lond. A 453 (1997), pp. 1611-1630.

[32] A. A. Wheeler, G. B. McFadden, and W. J. Boettinger, Phase-field model for solidification of a eutectic alloy, Proc. Roy. Soc. Lond. A 452 (1996), pp. 495-525.

Harald Garcke and Barbara Stoth: Institut für Angewandte Mathematik, WegelerStr. 6, D-53115 Bonn, Germany; email: harald@iam.uni-Bonn.de, Bstoth@iam.uni-Bonn.de

Britta Nestler: Foundry Institute, RWTH Aachen, Intzestr. 5, D-52072 Aachen, GerMANY; E-MAIL: BRITTA@GI.RWTH-AACHEN.DE 